\title{
Comment on Marciano et. al.: Effects of type 2 diabetes mellitus on coronary microvascular function and myocardial perfusion in patients without obstructive coronary artery disease
}

\author{
Luca Presotto
}

Received: 14 June 2012 / Accepted: 29 August 2012 /Published online: 3 November 2012

(C) Springer-Verlag Berlin Heidelberg 2012

Dear Sir,

I recently read with interest the article by Marciano et al. about the effect of diabetes mellitus on coronary microvascular diseases. I was quite puzzled by the analysis of the data shown in Fig. 2.

The author states in the results that there is a significant correlation between CPT-CF and fasting glycaemia. Visual inspection of the graphs would appear to reveal a different conclusion, as the two quantities look disconnected. Anscombe [1] explained the importance of looking not only at the correlation coefficient value but also at the data distribution. He provided, as examples, four different datasets, each characterized by the same mean and variance along $X$ and $Y$, the same regression line and the same (extremely statistically significant) correlation coefficient ( 0.816 with $n=11)$. In only one of these four examples were the correlation coefficient and the regression line indeed indicative of a "correlation" as commonly understood. Specifically, the fourth dataset shows a situation representative of the situation shown in Fig. 2 of the aforementioned article [2]. Anscombe shows that taking ten points with no correlation at all and one outlier very far from all the other ones gives a very high correlation coefficient. This, of course, is not indicative of a relationship, nonetheless linear, between the variables! A more formal analysis could reveal clearly the mathematical hypothesis that is violated when computing a correlation coefficient and its $p$ value from data with such a distribution.

An author's reply to this comment is available at doi 10.1007/s00259012-2252-3.

L. Presotto $(\bowtie)$

University of Milan Bicocca,

Milan, Italy

e-mail: presotto.luca@hsr.it
Therefore, I would say that the statement that "a significant inverse correlation was found between fasting glycaemia and endothelium-dependent vasodilatation" is not correct. In fact the analysis of only diabetic patients does not support this finding. With the exception of a small number of outliers (4 out of 49 total patients), all of the points seems to lie inside a perfect circle, the very shape used to describe uncorrelated variables.

If the point corresponding to the patient with a fasting glycaemia of $300 \mathrm{mg} / \mathrm{dl}$ and a CPT-CF ratio of about 1.3 were removed the correlation coefficient would drop to about zero. That would also happen with a reduction of fasting glycaemia and an increase in CPT-CF ratio, within the boundaries of the precision of the two measurements. The fasting glycaemia values and CPT-CF ratios of the three other "outlier" points suggest that these probably represent just the three most healthy nondiabetic patients.

\section{References}

1. Anscombe FJ. Graphs in statistical analysis. Am Stat. 1973;27 (1):17-21. JSTOR 2682899.

2. Marciano C, Galderisi M, Gargiulo P, Acampa W, D'Amore C, Esposito R, et al. Effects of type 2 diabetes mellitus on coronary microvascular function and myocardial perfusion in patients without obstructive coronary artery disease. Eur J Nucl Med Mol Imaging. 2012;39:1199-206. doi:10.1007/s00259-012-2117-9. 